\title{
Non-standard numerical scheme for singularly perturbed parabolic partial differential equation with long time lag arising in control theory
}

\author{
NAOL NEGERO ${ }^{1}$ and Gemechis Duressa ${ }^{2}$ \\ ${ }^{1}$ Wollega University \\ ${ }^{2}$ Jimma University
}

July 23, 2021

\begin{abstract}
For the numerical solution of singularly perturbed second-order parabolic partial differential equation of one dimensional convection-diffusion type with long time delays arising in control theory, a novel class of fitted operator finite difference methods is constructed using non-standard finite difference methods. Since the two parameters; time lag and perturbation parameters are sources for the simultaneous occurrence of time-consuming and high speed phenomena of the physical systems that depends on the present and past history, our study here is to capture the effect of the two parameters on the boundary layer. The spatial derivative is suitably replaced by a difference operator followed by the time derivative is replaced by the Crank-Nicolson based scheme. A second-order parameter-uniform error bounds are established to provide numerical results.
\end{abstract}

\section{Hosted file}

Naol-MMAS2021.pdf available at https://authorea.com/users/427184/articles/531413-nonstandard-numerical-scheme-for-singularly-perturbed-parabolic-partial-differentialequation-with-long-time-lag-arising-in-control-theory 